\title{
Therapeutic Implication of Human Bone Marrow Mesenchymal Stem Cell-Conditioned Medium to Reduce Cystogenic Potential of CD133+ Renal Progenitor Cells of Human Polycystic Kidneys
}

\author{
Ehsan Ehsani ${ }^{1,2}$, Sara Hajibabaei ${ }^{1}$, Farid Dadkhah ${ }^{3,4}$ and Reza \\ Moghadasali ${ }^{1 *}$ \\ ${ }^{1}$ Department of Stem Cells and Developmental Biology, Cell Sciences Research Center, Royan \\ Institute for Stem Cell Biology and Technology, ACECR, Tehran, Iran \\ ${ }^{2}$ Department of Biology, Roudehen Branch, Islamic Azad University, Roudehen, Iran
}

${ }^{3}$ Department of Andrology, Reproductive Biomedicine Research Center, Royan Institute for

\section{Investigation Paper}

Volume 4 Issue 1

Received Date: January 06, 2020

Published Date: February 14, 2020

DOI: $10.23880 /$ jes-16000135 Reproductive Biomedicine, ACECR, Tehran, Iran

${ }^{4}$ Department of Surgical Urology, Modarres Hospital, Shahid Beheshti University of Medical Sciences, Tehran, Iran

*Corresponding author: Reza Moghadasali, Department of Stem Cells and Developmental Biology, Cell Sciences Research Center, Royan Institute for Stem Cell Biology and Technology, ACECR, Tehran, Iran, Tel: +982122306485; Fax: +982123562507; Email: Rezamoghadasali@Royaninstitute.org

\section{Abstract}

Autosomal dominant polycystic kidney disease (ADPKD) is a genetic disorder in which, epithelial cells are somehow dedifferentiated but highly proliferative and form renal cysts. There are also proliferative CD133+ renal progenitor cells in an ADPKD kidney. Among several therapeutic strategies that exist to heal ADPKD, bone marrow mesenchymal stem cells (BMMSC) are of great interest. So, we examined human BM-MSC-condition medium (hBM-MSC-CM) effects on polycystic CD133+ renal progenitor cells. For this purpose, polycystic CD133+ renal progenitor cells were isolated from patients undergoing nephrectomy for kidney transplantation. Differentiation and cystogenic potentials of polycystic CD133+ renal progenitor cells were evaluated using hBM-MSC-CM. Polycystic-lining cells expressed CD133 as a renal progenitor cell marker. These cells were more proliferative and presented a defective epithelial differentiation phenotype compared to normal kidney CD133+ renal progenitor cells. Also, they were not able to express differentiated tubular epithelial cell markers such as E-cadherin and ZO-1. Moreover, polycystic CD133+ renal progenitor cells, in contrast to normal CD133+ renal progenitor cells, formed cysts in a three-dimensional (3D) culture system. hBM-MSC-CM treatment decreased proliferation and cystogenesis, but increased differentiation of polycystic CD133+ renal progenitor cells in vitro. All in all, hBM-MSC-CM could decrease the proliferation and cystogenesis through induction of differentiation in polycystic CD133+ renal progenitor cells.

Keywords: Autosomal Dominant Polycystic Kidney Disease; CD133+ Renal Progenitor Cells; Bone Marrow Mesenchymal Stem Cells; Cystogenesis 


\section{Journal of Embryology \& Stem Cell Research}

Abbreviations: ADPKD: Autosomal Dominant Polycystic Kidney Disease; BM-MSC: Bone Marrow Mesenchymal Stem Cells; PKDs: Polycystic Kidney Diseases; ESRD: End-Stage Renal Disease; BM-SCs: Bone Marrow-Derived Stem Cells; CKD: Chronic Kidney Disease; AKI: Acute Kidney Injury; PBS: Phosphate-Buffered Saline; FACS: Fluorescence-Activated Cell Sorting; HGF: Hepatocyte Growth Factor; SD: Standard Deviation; CFSC: Carboxyfluorescein Succinimidyl Ester; ARPKD: Autosomal Recessive Polycystic Kidney Disease; TKV: Total Kidney Volume; ACE: Angiotensin-Converting Enzyme.

\section{Introduction}

Autosomal dominant polycystic kidney disease (ADPKD) is a multifactorial disorder with several genetic variants. The disorder is characterized by the appearance of numerous cysts in the kidneys. ADPKD is $85 \%$ of polycystic kidney diseases (PKDs), with an incidence of 1:1000 subjects. Onset of the disease is after 20-35 years of age. The disease is due to mutations in the short arm of Ch. 16 (PKD1) and approximately $50 \%$ of patients develop end-stage renal disease (ESRD) between the fifth and sixth decade of life [1].

The mechanisms of cellular and molecular cystogenesis have remained unclear. However, it seems that proliferative cyst-lining cells that express the stem cell marker, CD133 as a marker of renal progenitor cells within the nephron, both in cortex and inner medulla, show features of nondifferentiated mesenchymal progenitors [2]. In addition, CD133 may be considered a marker of renal repair, as the hypoxic microenvironment occurring after injury may favor the acquirement of CD133 renal progenitor properties by nephron cells [3]. CD133 expression in renal pathology was found to be related to excessive proliferation and/or reduced differentiation of renal progenitors, which occurs in polycystic kidney disease and glomerular diseases. Also, CD133 may not be considered a promising marker for renal cancer stem cells [4-6]. Besides, CD133 sounds like a potential therapeutic target for nonmalignant renal pathology such as PKD [3]. Under pathological conditions, an increased number of CD133+ cells was observed. CD133+ cells contribute to the formation of glomerular crescents and line the cysts in the polycystic kidney disease [2,7]. Therefore, a deregulated CD133+cell proliferation or differentiation may be involved in glomerular and tubular response to injury under pathological condition [8].

Lodi and colleagues showed CD133 and CD24 expression in renal tissue of patients affected by ADPKD [9]. Carvalhosa and colleagues reported that $\mathrm{CD} 133$ + renal progenitor cells exist in ADPKD lining cysts [2].

CD133 is expressed by cells lining the Bowman capsule, proximal tubules and ascending limb of the Henle's loop [1013]. In renal pathology, such cells may participate in crescent formation in glomeruli [7]. In PKD, CD133+ cells line the wall of cysts [2]. Hypoxia stimulates stem-related functions and the stem transcription factor Oct4 and increases CD133 expression [3]. So, selective targeting of CD133 may allow pharmacological interventions to halt proliferation or induce differentiation of CD133+ cells.

Moreover, bone marrow-derived stem cells (BM-SCs), particularly bone marrow-derived mesenchymal stem cells (BM-MSCs), may also contribute to renal repair. However, we know little about their therapeutic effects. Some studies suggested cell fusion between BM and resident tubular cells $[14,15]$. Other studies documented that BM-SCs are able to differentiate in resident cells [16], while some proposed that BM-SCs facilitate organ regeneration by a paracrine action (i.e. via secreting growth factors such as HGF, VEGF and IGF1) [17]. All these secreted molecules would provide a regenerative milieu able to constrain renal damage. These paracrine mechanisms can be as diverse as stimulating receptor mediated survival pathways including anti-apoptosis, and stem cell homing and differentiation pathways or those regulating the anti-inflammatory effects in damaged areas.

Therapeutic potential of MSCs is not limited to anti-fibrotic and anti-inflammatory effects on chronic kidney disease (CKD) and acute kidney injury (AKI) but they are also applicable for controlling PKD progress [18].

In this regard, Franchi and colleagues documented that MSCs improve reno-vascular function in PKD, reduce fibrosis and preserve the expression of the pro-angiogenic molecules, but do not affect cyst size and number [19].

Accordingly, the effects of MCSs in patients with ADPKD were recently evaluated in a clinical trial phase I with a 12month follow-up; results approved the safety and tolerability of an intravenous (i.v.) injection of autologous BM-MSCs [20]. Also, we previously noted that kidney-derived MSCs induced differentiation of CD133+ renal progenitor cells derived from embryonic stem cell toward renal epithelial cells [21].

It was reported that several processes and pathways are involved in ADPKD development, such as apoptosis, fibrosis, inflammation, cyst proliferation, renin-angiotensin system (RAS) activation, and kidney vasculature impairment [19]; MSCs may affect these pathways through different mechanisms [22-27].

Thus, we herein examined cystic disease inhibition and loss of renal function attenuation through decreasing proliferation and increasing differentiation in cystic and 


\section{Journal of Embryology \& Stem Cell Research}

non-cystic tubules cells by hBM-MSC-CM.

\section{Materials and Methods}

\section{Kidney Sampling, Tissue Processing and Immunohistochemistry}

We performed genetic, ultrasound, MRI imaging and paraclinical assessments on ADPKD patients as well as healthy subjects.

Normal kidney samples were isolated by autopsy from human cadavers $(\mathrm{N}=3)$ within $12-24$ hours after death. PKD samples were isolated by biopsy from ADPKD patient $(\mathrm{N}=1)$ during kidney transplantation and nephrectomy. All experimental procedures involving human participants were done in accordance with the ethical standards of the Ethics Committee of Royan Institute and the Institutional Review Board (No. EC.93.1005). Then, samples were transferred into a sterile tube containing phosphate-buffered saline (PBS) (Gibco, USA) and 1\% penicillin-streptomycin (pen/strep; Gibco, USA). The sampling tubes were kept on the ice and transferred to tissue culture lab. Tissues were divided into two parts. Samples of the first part were used for cell culture and isolation of CD133+ renal progenitor cells and those of the second part were washed twice with PBS, fixed with $4 \%$ paraformaldehyde for $24 \mathrm{~h}$ at $4^{\circ} \mathrm{C}$ and used for histological analysis. The samples were then dehydrated through a series of graded alcohol solutions and xylol and embedded in paraffin. The paraffin-embedded specimens were sectioned into $5-\mu \mathrm{m}$ thickness, placed on poly-l-lysine-coated glass slides and placed in an oven at a temperature of $60^{\circ} \mathrm{C}$ for 12 $\mathrm{h}$; then, they were deparaffinized and dewaxed in xylene, stained with hematoxylin and eosin (H\&E) and observed by means of light microscopy. For CD133 staining, samples were incubated with CD133 (1:100) (Miltenyi Biotec, USA) antibody conjugated with rat anti-mouse IgG1-fluorescence isothiocyanate (FITC).

\section{Renal Progenitor Cells Culture, Sorting And Isolation}

Kidney samples were digested using collagenase type I (Sigma, USA) for 1 hour at $37^{\circ} \mathrm{C}$ and then washed twice with PBS and filtered through filter mesh $(70 \mu \mathrm{m})$. A singlecell suspension was incubated in low-glucose DMEM medium (Gibco, USA) supplemented with 10\% FBS (Gibco, USA).

We used fluorescence-activated cell sorting (FACS) for isolating CD133+ renal progenitor cells. We harvested the cultured cells form normal kidney and PKD cells into a singlecell suspension using trypsin/EDTA, washed the cells twice with PBS, and re suspended them in blocking solution (PBS with $2 \%$ bovine serum albumin (BSA, Sigma, Germany)) for
$30 \mathrm{~min}$. Then, we incubated cells with phycoerythrin (PE)conjugated mouse anti-human CD133 (1:100, Miltenyi Biotec) for 30 min at $4^{\circ} \mathrm{C}$ in the dark. Next, cells were washed twice with PBS. Cells incubated with identical concentrations of PE, and conjugated mouse IgG isotype antibodies $11: 200$, Miltenyi Biotec; 1:200, BD Pharmingen), served as the negative controls. Finally, we performed cell sorting and analysis by BD FACSAria (BD Bioscience, San Jose, CA), and FlowJo software (version 7.6.1), respectively.

\section{CD133+Renal Progenitor Cells Characterization}

Surface markers of cultured cells were analyzed using fluorescence-activated cell sorting by BD FACSAria. For immune phenol typing, cells were dissociated in $0.05 \%$ trypsin-EDTA (Gibco, USA) and washed in PBS (Gibco, USA) supplemented with $1 \%$ heat-inactivated FBS (Hyclone, USA) and 2 mM EDTA (Merck, Darmstadt, Germany). Next, 4-5 × 10 ${ }^{5}$ cells were incubated with primary antibodies for detection of surface markers, for two hours and with the secondary antibodies for 30 minutes, both done at $4^{\circ}$ C. Surface markers that were analyzed included VEGFR2 (1:200) (BD Pharmingen, USA) conjugated with PE-Mouse IgG1k (BD PharmingenTM, Cat NO: 551436) and PE-conjugated mouse anti-human CD133 (1:100) (Miltenyi Biotec), as well as CD34 and CD45 (BD Pharmingen) conjugated with FITC. Controls samples were stained with appropriate isotypematched antibodies. Flow cytometry analysis was performed in triplicate using BD FACS Calibur Flow Cytometer (BD Biosciences, Franklin Lakes, NJ, USA). Data was analyzed by FlowJo software (version 7.6.1).

\section{Human BM-MSC-Conditioned Medium (CM) Preparation}

BM-MSCs were isolated from healthy donors at Royan Institute, as described before [28]. In short, MSCs were isolated from each BM sample by Ficoll density gradient centrifugation (Lymphodex, Inno-TRAIN, REF: 002041600). Afterwards, the cells were seeded into polystyrene culture flasks (BD, USA) at a density of $1 \times 10^{6}$ cells $/ \mathrm{cm}^{2}$ in alphaMinimum Essential Medium (alpha MEM), with $100 \mathrm{U} /$ $\mathrm{ml}$ penicillin, $0.1 \mathrm{mg} / \mathrm{ml}$ streptomycin (Gibco BRL), $2 \mathrm{mM}$ L-glutamine (Gibco) and 10\% FBS (Hyclone). Cultures, maintained in a humidified atmosphere with $5 \%(\mathrm{v} / \mathrm{v}) \mathrm{CO}_{2}$ at $37^{\circ} \mathrm{C}$, had their medium changed twice weekly thereafter. On reaching $60-80 \%$ confluency, adherent cells were detached after treatment with trypsin/EDTA solution (0.2\%) (Gibco) for re-seeding at $10^{3}$ cells per $/ \mathrm{cm}^{2}$. To obtain hBM-MSC$\mathrm{CM}$, cells were cultured in DMEM medium containing low glucose $(1 \mathrm{~g} / \mathrm{l})$, supplemented with penicillin $(100 \mathrm{U} / \mathrm{ml})$, streptomycin $(100 \mu \mathrm{g} / \mathrm{ml})$ and $10 \%$ FBS. We cultured human BM-MSCs to reach the desired confluency and collected the medium after approximately 24-48 $\mathrm{h}$ after refreshing 


\section{Journal of Embryology \& Stem Cell Research}

it. hBM-MSC-CM was centrifuged at $1500 \mathrm{rpm}$ for $5 \mathrm{~min}$ to remove detached MSCs and stored at $-80 \circ \mathrm{C}$ until further use. hBM-MSC-CM was diluted (1:1) in sorted-CD133+ renal progenitor cells culture medium prior to incubation.

\section{CD133+ Renal Progenitor Cells Treatment with hBM-MSC-CM}

Sorted polycystic CD133+ renal progenitor cells were cultured in hBM-MSC-CM and compared with sorted polycystic CD133+ renal progenitor cells and sorted normal CD133+ renal progenitor cells. Control groups were cultured in REGM medium (PromoCell) including 10\%FBS + hepatocyte growth factor (HGF) (10 ng/ml, Sigma) and fibroblast growth factor-4 (FGF-4) $(10 \mathrm{ng} / \mathrm{ml}$, Sigma).

\section{Immunocytochemical Staining}

Sorted-CD133+ renal progenitor cells were washed twice with PBS and fixed with $4 \%$ paraformaldehyde at 4을 overnight, before and after treatment with hBM-MSC-CM. The cells were permeabilized and blocked in PBS containing 0.2\% Triton X-100 for $10 \mathrm{~min}$ and $10 \%$ goat serum for $30 \mathrm{~min}$ and then incubated in diluted primary antibody overnight. The antibodies used in this study were anti-cytokeratin 8 (CK8)
(Chemicon, USA), E-cadherin (BD, USA) and ZO-1 (BD, USA). At the end of the incubation period, the cells were washed twice with PBS+ $0.05 \%$ tween 20 and incubated with PE and FITC-conjugated anti-mouse IgG (BD, USA) diluted in $0.5 \%$ BSA at $4{ }^{\circ} \mathrm{C}$ overnight. After washing twice with $\mathrm{PBS}+0.05 \%$ tween 20, specimens were examined under fluorescence microscope (BX51, Olympus, Japan).

\section{Statistical Analysis}

Data analysis was performed using GraphPad Prism 7.03 (GraphPad Software, Inc.). Data are presented as mean \pm standard deviation (SD). Statistical analysis was performed by one-way ANOVA to determine significant differences between groups at $P<0.05$.

\section{Results}

\section{CD133+ Express in Polycystic-Lining Cells}

Biopsy of PKD tissues were stained with H\&E. We found cysts of different sizes (Figures 1A \& B). In PKD tissues, we found border staining for CD133 marker. In particular, all polycystic-lining cells highly expressed CD133 marker (Figures 1C \& D). However, we found almost no CD133 expression in normal kidney samples (Figure 1E-G).

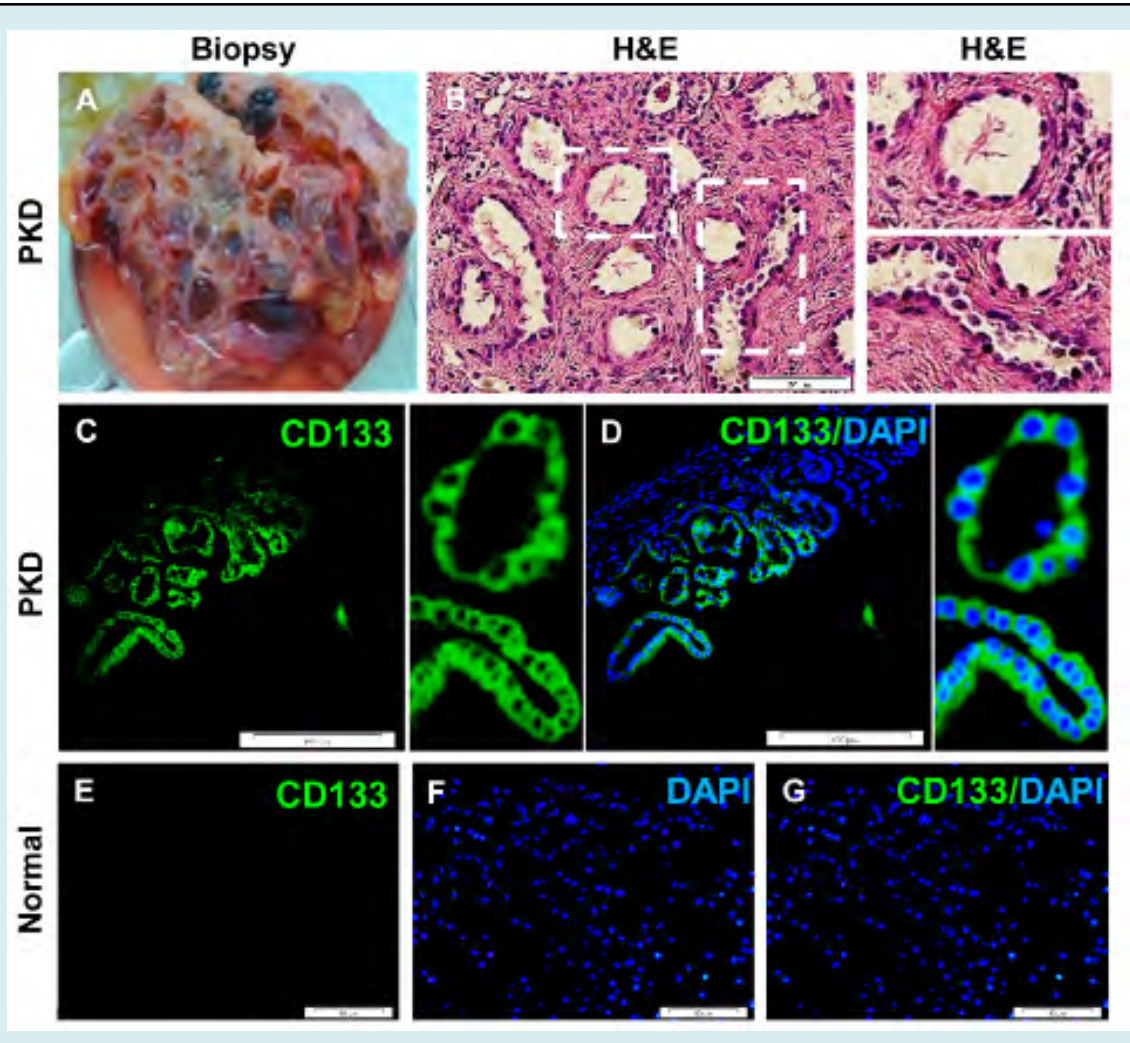

Figure 1: Histological evaluation of PKD and normal kidney tissues. A: PKD tissue biopsy, B: H\&E staining for PKD tissues, C \& D: CD133 expression in polycystic-lining cells, E \& G: normal kidney tubular cells without CD133 expression. 


\section{Polycystic CD133+ Renal Progenitor Cells were Proliferative}

We could isolate PKD and normal kidney cells. Cell cultured in expansion medium formed colonies in 1-3 days and reached optimized confluence in 7-10 days (Figures 2A-F). Cells isolated from PKD were proliferative and expressed CD133 while normal cells expressed CD133 sparsely. However, the endothelial marker VEGFR2 was not expressed by PKD cells, but the normal cells were positive for this marker. Nevertheless, haematopoietic markers, CD45 and CD34 were absent in both cell groups (Figures 2G-I). Proliferation potential was assessed by carboxyfluorescein succinimidyl ester (CFSE) fluorochromes staining in 7 days and analysed by flowcytometry (unpublished data). The proliferative count showed a higher proliferative potential for PKD cells compared to normal subjects (Figure 2J). The PKD cells were maintained in expansion medium for upto nine passages and they maintained CD133 expression, indicating a limited self-renewal for the cells.
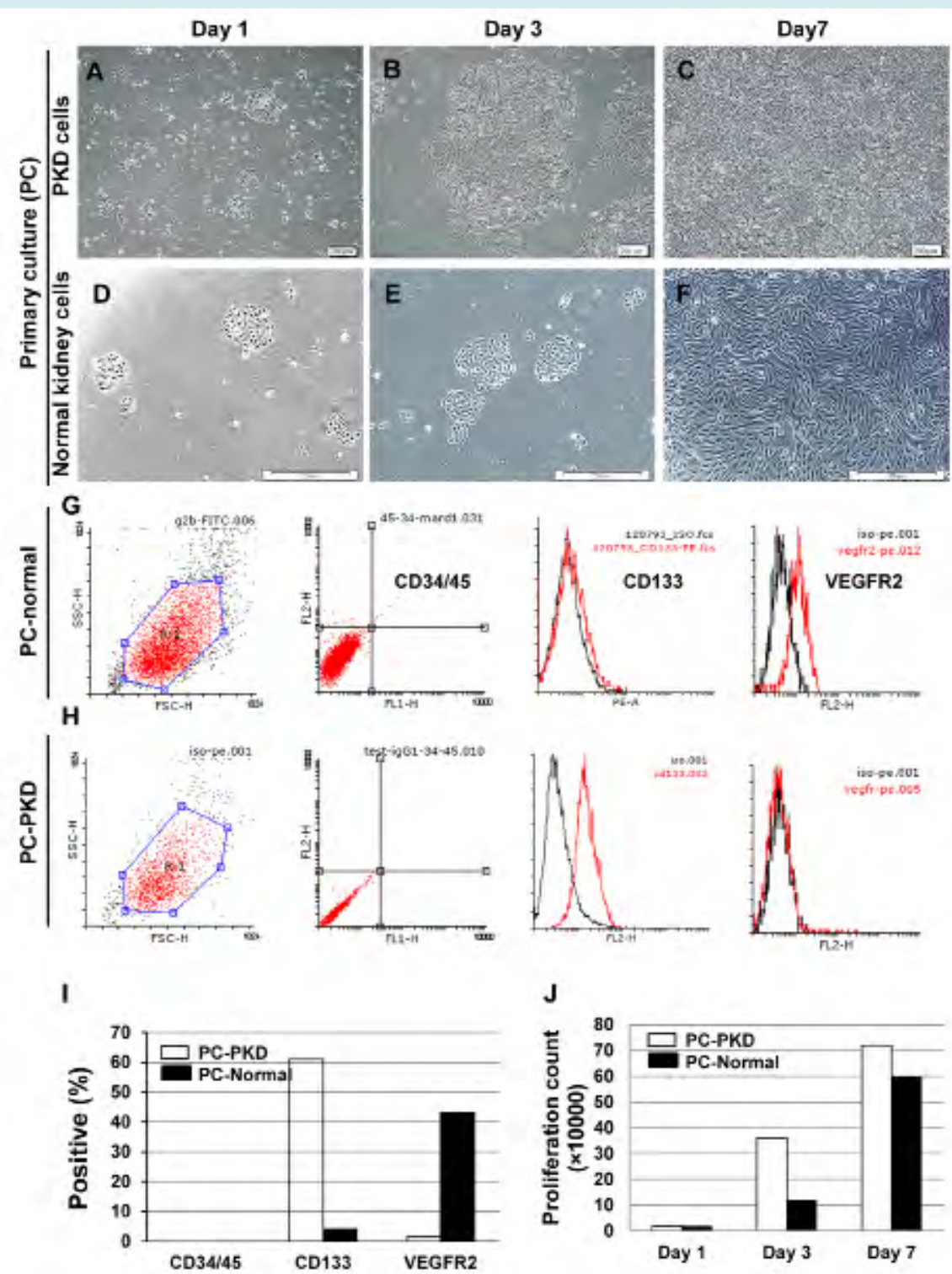

Figure 2: Polycystic CD133+ renal progenitor cells proliferation in culture. A-F: Colony formation and expansion of PKD and normal cells after 7 days. G-I: Cells isolated from PKD were proliferative and expressed CD133 in comparison with normal cells. However, the endothelial marker VEGFR2 was not expressed by PKD cells, but expressed by normal cells. Haematopoietic markers CD45 and CD34 were absent in both of them. J: Proliferative count showed that PKD cells had a higher proliferative potential compared to normal cells. 


\section{Polycystic CD133+ Renal Progenitor Cells Showed Low Adherent Junctions}

CD133+ renal progenitor cells were very low and undetectable in normal kidney tissues. However, they proliferate in culture after isolation from tissue in vitro. So, sorted CD133+ renal progenitor cells were isolated from 2D culture of PKD and normal cells were sorted by FACS.

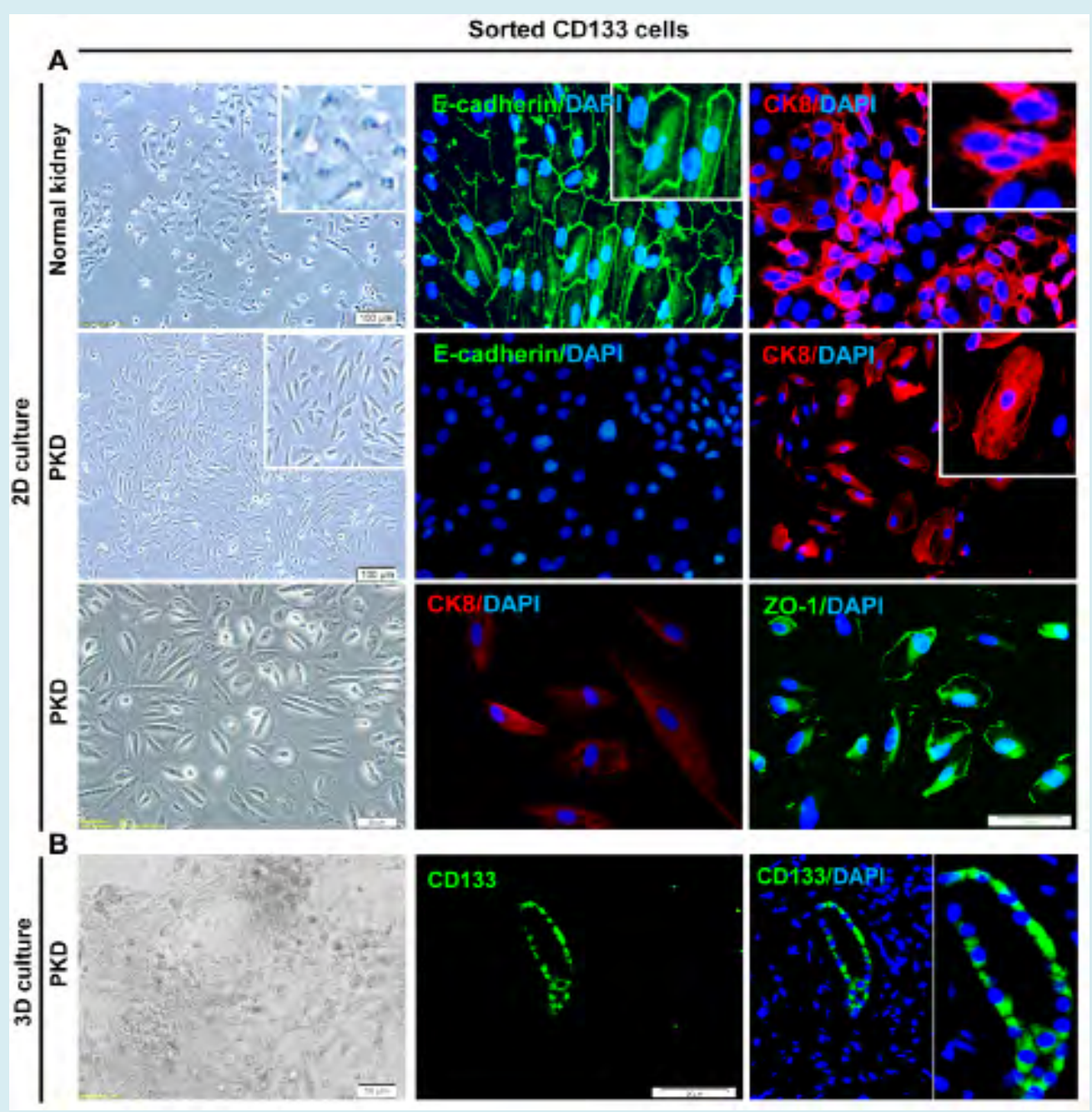

Figure 3: Sorted polycystic CD133+ renal progenitor cells with low adherent junctions' expression. A: Sorted polycystic and normal CD133+ renal progenitor cells were positive for CK8. However, sorted polycystic CD133+ renal progenitor cells were negative for E-cadherin. ZO-1 was expressed at low levels in sorted polycystic CD133+ renal progenitor cells. But, normal CD133 progenitor cells were positive for E-cadherin and ZO-1. B: Sorted polycystic CD133+ renal progenitor cells were positive for CD133 marker in 3D culture on collagen type I.

Sorted PKD CD133+ renal progenitor cells were positive for CK8, similar to normal CD133+ renal progenitor cells. However, sorted PKD CD133+ renal progenitor cells did not express E-cadherin and ZO-1. Nevertheless, normal CD133 progenitor cells expressed both E-cadherin and Z0-1 markers (Figure 3A). In 3D culture, sorted PKD CD133+ renal progenitor cells seeded on collagen type I, expressed CD133 marker (Figure 3B).

\section{CD133+ Renal Progenitor Cells Showed Increased Adherent Junctions but Reduced Cystogenic Potential Upon Hbm-MSC-CM Treatment}

Sorted polycystic CD133+ renal progenitor cells were cultured in hBM-MSC-CM (Figure 4A). hBM-MSC-CM reduced cystogenic potential of polycystic CD133+ renal progenitor 


\section{Journal of Embryology \& Stem Cell Research}

cells, decreased the proliferation rate (Figure 4B) while increased adherent junctions (Figure 4C).

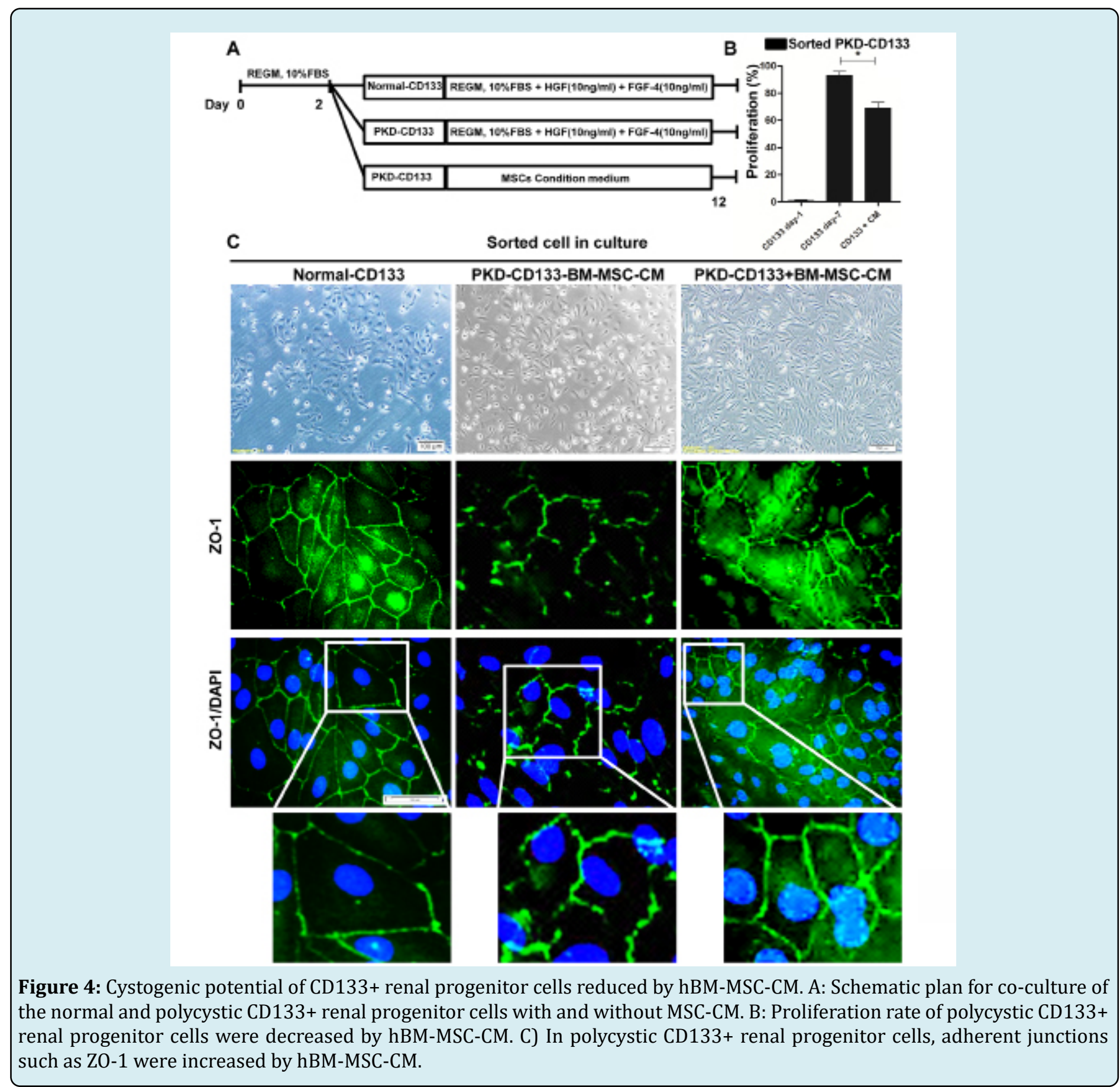

\section{Discussion}

CD133+ renal progenitor cell niches were identified in the renal papillae in animals as well as human urinary pole of the Bowman's capsule. Although these cells may contribute to organ regeneration, it remains uncertain how these cells exert this effect and participate in healing process upon kidney injury. Nevertheless, renal progenitor cells may be therapeutic tools for treatment of renal diseases.

In ADPKD, cysts grow focally and disrupt normal renal tissue, leading to renal failure. Carvalhosa and colleagues 


\section{Journal of Embryology \& Stem Cell Research}

showed that cyst-lining cells express the renal progenitor cells marker CD133 [2]. CD133+ renal progenitor cells isolated from polycystic kidney, carry mutations in PKD genes and show a dedifferentiated phenotype, similar to $\mathrm{CD} 133+$ renal progenitor cells from normal kidney [2]. Also, these cells are more proliferative and present a defective epithelial differentiation phenotype compared to normal renal CD133+ cells as reflected by their inability to express all tubular epithelial cell markers when cultured in epithelial differentiation medium [2]. We demonstrated that polycystic CD133+ renal progenitor cells were negative for epithelial marker, E-cadherin and had a low expression of ZO-1, a tight junction marker. Polycystic CD133+ renal progenitor cells formed cysts in vitro in a 3D culture system and in vivo when injected subcutaneously within Matrigel in SCID mice in contrast to normal kidney CD133+ renal progenitor cells [2]. Rapamycin treatment reduced in vitro proliferation of polycystic CD133+ renal progenitor cells and decreased cystogenesis both in vitro and in vivo [2]. The in vitro epithelial differentiation was only partially improved by rapamycin. These results indicated that polycystic CD133+ renal progenitor cells retained a dedifferentiated phenotype and were able to produce cysts [2].

Therapeutic implications might involve conjugating the CD133 antibodies with different types of medications to target CD133+ renal progenitor cells in nonmalignant renal pathology. As described above, CD133+ renal progenitor cells in cysts of polycystic kidney are characterized by an increased proliferation rate and reduced differentiation. It seems to be of great value in pharmacological approaches to increase their differentiation.

On the other hand, a balanced cellular proliferation and programmed cell death is essential for normal growth and differentiation of the kidney cells. These fundamental processes are disturbed in polycystic kidneys. In both ADPKD and autosomal recessive polycystic kidney disease (ARPKD), abnormal apoptosis is observed and can destroy normal renal parenchyma, allowing cystic epithelia to proliferate. The importance of apoptosis was highlighted in knockout mouse, where inactivation of inhibitors of apoptosis (bcl-2 or activating protein $2 b$ [AP-2b]) leads to development of cystic kidney disease.

However, PKD cystogenesis could be affected by different signaling pathways. In this regard, MAPK signaling has an important role in the pathogenesis of ADPKD via increment of proliferation [1]. Studies indicated that MSC-CM has anti-apoptotic effects mediated through suppressing P38 MAPK and ERK expression in an AKI rat model [29]. MSCs suppressed NF-kB pathway [30] and pro-inflammatory cytokines, and blocked inflammation in a CKD model [31]. Human MSCs seems to be appropriate for angiogenesis through VEGF secretion [19]. Reports demonstrated that RAS inhibition improves blood pressure (BP) and total kidney volume (TKV), and prevents cyst development in animal and clinical models [32,33]. MSCs reduce renin, angiotensinconverting enzyme (ACE), and angiotensin II type 1 (AT1) receptor, and decrease $\mathrm{BP}$, inflammation, and fibrosis [34,35]. The MSCs inhibitory effect on RAS is more stable than that of ACE inhibitors [34]. Ehsani and colleagues investigated the impact of kidney-derived MSCs on differentiation of CD133+ renal progenitor cells derived from embryonic stem cell into epithelial tubular cells. They showed that embryoid-derived CD133+ cells expressed renal progenitor cells marker genes but not hematopoietic stem cell and renal epithelial markers. They reported that CD133+ renal progenitor cells expressed renal epithelial markers namely, CK8, CK18, ZO1 and E-Cadherin after being co-cultured with kidney derived MSCs [21]. Therefore, the MSCs features make them applicable for PKD treatment. We herein showed that hBM-MSC-CM may increase $\mathrm{ZO}-1$ but decrease proliferation rate in polycystic CD133+ renal progenitor cells.

\section{Conclusion}

Kidney polycystic CD133+ renal progenitor cells retain a dedifferentiated phenotype with high proliferative potency as well as cysts generating capacity. In addition, hBM-MSC$\mathrm{CM}$ can enhance the differentiation potential and reduce the proliferation and cystogenesis potential of polycystic CD133+ renal progenitor cells. We showed that hBM-MSC$\mathrm{CM}$ is an appropriate candidate for suppressing the cysts progress in ADPKD patients.

\section{Acknowledgment}

We would like to particularly thank to Dr. Mohammad Reza Ghadirzadeh, Dr. Ali Reza Kadkhodaei and Dr. Ali Ziaie in Legal Medicine Research Center, Iranian Legal Medicine Organization, Tehran, Iran for their help with tissue sampling.

\section{Funding}

This study was supported by a grant from Royan Institute and the Royan Charity Association for Health Research (Tehran, Iran).

\section{Authors' Contributions}

E. E, S.H. and R.M.: Idea and study design, collection and assembly of data, data analysis and interpretation, and manuscript writing. F.D.: Tissue sampling and collection. E. E, R.M. and S.H.: Collection and assembly of data. R.M.: Final approval of the manuscript. R.M.: Financial support. 


\section{Journal of Embryology \& Stem Cell Research}

\section{Conflict of Interest}

The authors have no conflicts of interest.

\section{References}

1. Malekshahabi T, Rad NK, Serra Al, Moghadasali $R$ (2019) Autosomal dominant polycystic kidney disease: Disrupted pathways and potential therapeutic interventions. Journal of cellular physiology 234(8): 12451-12470.

2. Carvalhosa R, Deambrosis I, Carrera P, Pasquino C, Rigo F, et al. (2011) Cystogenic potential of CD133+ progenitor cells of human polycystic kidneys. The Journal of pathology 225(1): 129-141.

3. Bussolati B, Collino F, Camussi G (2012) CD133+ cells as a therapeutic target for kidney diseases. Expert opinion on therapeutic targets 16(2): 157-165.

4. Bruno S, Bussolati B, Grange C, Collino F, Graziano ME, et al. (2006) CD133+ renal progenitor cells contribute to tumor angiogenesis. The American journal of pathology 169(6): 2223-2235.

5. Addla SK, Brown MD, Hart CA, Ramani VAC, Clarke NW, et al. (2008) Characterization of the Hoechst 33342 side population from normal and malignant human renal epithelial cells. American Journal of Physiology-Renal Physiology 295(3): F680-F687.

6. Goodell MA, Brose K, Paradis G, Conner AS, Mulligan RC (1996) Isolation and functional properties of murine hematopoietic stem cells that are replicating in vivo. Journal of Experimental Medicine 183(4): 1797-1806.

7. Smeets B, Angelotti MC, Rizzo P, Dijkman H, Lazzeri E, et al. (2009) Renal progenitor cells contribute to hyperplastic lesions of podocytopathies and crescentic glomerulonephritis. Journal of the American Society of Nephrology 20(12): 2593-2603.

8. Bussolati B, Camussi G (2013) New insights into the renal progenitor cells and kidney diseases by studying CD133, in Prominin-1 (CD133): New Insights on Stem \& Cancer Stem Cell Biology. Springer pp: 113-123.

9. Lodi D, Ligabue G, Cavazzini F, Lupo V, Cappelli G, et al. (2013) CD133 and CD24 expression in renal tissue of patients affected by autosomal dominant polcystic kidney disease. 3-4: 211-217.

10. Bussolati B, Bruno S, Grange C, Buttiglieri S, Deregibus $\mathrm{MC}$, et al. (2005) Isolation of renal progenitor cells from adult human kidney. The American journal of pathology
166(2): 545-555.

11. Sagrinati C, Netti GS, Mazzinghi B, Lazzeri E, Liotta F, et al. (2006) Isolation and characterization of multipotent progenitor cells from the Bowman's capsule of adult human kidneys. Journal of the American Society of Nephrology 17(9): 2443-2456.

12. Sallustio F, Benedictis LD, Castellano G, Zaza G, Loverre A, et al. (2010) TLR2 plays a role in the activation of human resident renal stem/progenitor cells. The FASEB Journal 24(2): 514-525.

13. Bussolati B, Moggio A, Collino F, Aghemo G, D'Armentoet G, et al. (2011) Hypoxia modulates the undifferentiated phenotype of human renal inner medullary CD133+ progenitors through Oct $4 / \mathrm{miR}-145$ balance. American Journal of Physiology-Renal Physiology 302(1): F116-F128.

14. Fang TC, Alison MR, Cook HT, Jeffery R, Wright NA, et al. (2005) Proliferation of bone marrow-derived cells contributes to regeneration after folic acid-induced acute tubular injury. Journal of the American Society of Nephrology 16(6): 1723-1732.

15. Held PK, Al-Dhalimy M, Willenbring H, Akkari Y, Jiang S, et al. (2006) In vivo genetic selection of renal proximal tubules. Molecular Therapy 13(1): 49-58.

16. Poulsom R, Alison MR, Cook T, Jeffery R, Ryan E, et al. (2003) Bone marrow stem cells contribute to healing of the kidney. Journal of the American Society of Nephrology 14(S1): 48-54.

17. Spees JL, Lee RH, Gregory CA (2016) Mechanisms of mesenchymal stem/stromal cell function. Stem cell research \& therapy 7(1): 125.

18. Patel DM, Shah J, Srivastava AS (2013) Therapeutic potential of mesenchymal stem cells in regenerative medicine. Stem cells international 2013: 15.

19. Franchi F, Peterson KM, Xu R, Miller B, Psaltis PL, et al. (2015) Mesenchymal stromal cells improve renovascular function in polycystic kidney disease. Cell transplantation 24(9): 1687-1698.

20. Makhlough A, Shekarchian S, Moghadasali R, Einollahi B, Hosseini SE, et al. (2017) Safety and tolerability of autologous bone marrow mesenchymal stromal cells in ADPKD patients. Stem cell research \& therapy 8(1): 116.

21. Ehsani E, Shekarchian S, Baharvand H, Aghdami N, Moghadasali R, et al. (2019) Improved differentiation of human enriched CD133+CD24+renal progenitor cells derived from embryonic stem cell with embryonic 


\section{Journal of Embryology \& Stem Cell Research}

mouse kidney-derived mesenchymal stem cells coculture. Differentiation 109: 1-8.

22. Riella C, Czarnecki PG, Steinman TI (2014) Therapeutic advances in the treatment of polycystic kidney disease. Nephron Clinical Practice 128(3-4): 297-302.

23. Grantham JJ, Chapman AB, Torres VE (2006) Volume progression in autosomal dominant polycystic kidney disease: the major factor determining clinical outcomes. Clinical journal of the american society of Nephrology 1(1): 148-157.

24. Nagao S, Yamaguchi T, Kusaka M, Maser RL, Takahashi $\mathrm{H}$, et al. (2003) Renal activation of extracellular signalregulated kinase in rats with autosomal-dominant polycystic kidney disease. Kidney international 63(2): 427-437.

25. Okumura Y, Sugiyama N, Tanimura S, Nishida M, Hamaoka $\mathrm{K}$, et al. (2009) ERK regulates renal cell proliferation and renal cyst expansion in inv mutant mice. Acta Histochem Cytochem 42(2): 39-45.

26. Saigusa T, Dang Y, Bunni MA, Amria MY, Steele SL, et al. (2015) Activation of the intrarenal renin-angiotensinsystem in murine polycystic kidney disease. Physiological reports 3(5): e12405.

27. Torres VE, Donovan KA, Scicli G, Holley KE, Thibodeau SN, et al. (1992) Synthesis of renin by tubulocystic epithelium in autosomal-dominant polycystic kidney disease. Kidney international 42(2): 364-373.

28. Makhlough A, Shekarchian S, Moghadasali R, Einollahi B, Dastgheib M, et al. (2018) Bone marrow-mesenchymal stromal cell infusion in patients with chronic kidney disease: A safety study with 18 months of follow-up. Cytotherapy 20(5): 660-669.
29. Qi S, Wu D (2013) Bone marrow-derived mesenchymal stem cells protect against cisplatin-induced acute kidney injury in rats by inhibiting cell apoptosis. International Journal of Molecular Medicine 32(6): 1262-1272.

30. Wu HJ, Yiu WH, Li EX, Wong DWL, Leung JCk, et al. (2014) Mesenchymal stem cells modulate albumin-induced renal tubular inflammation and fibrosis. PLoS One 9(3): e90883.

31. Semedo P, Correa-Costa M, Cenedeze MA, Malheiros DMAC, Antonia dos Reis M, et al. (2009) Mesenchymal stem cells attenuate renal fibrosis through immune modulation and remodeling properties in a rat remnant kidney model. Stem cells 27(12): 3063-3073.

32. Schrier RW, Abebe KZ, Perrone RD, Torres VE, Braun WE, et al. (2014) Blood pressure in early autosomal dominant polycystic kidney disease. New England Journal of Medicine 371(24): 2255-2266.

33. Zafar I, Tao Y, Falk S, McFann K, Schrier RW, et al. (2007) Effect of statin and angiotensin-converting enzyme inhibition on structural and hemodynamic alterations in autosomal dominant polycystic kidney disease model. American Journal of Physiology-Renal Physiology 293(3): F854-F859.

34. Gregorini M, Corradetti V, Rocca C, Pattonieri EF, Valsania T, et al. (2016) Mesenchymal stromal cells prevent renal fibrosis in a rat model of unilateral ureteral obstruction by suppressing the renin-angiotensin system via HuR. PLoS One 11(2): e0148542.

35. Oliveira-Sales EB, Maquigussa E, Semedo P, Pereira LG, Ferreira VM, et al. (2013) Mesenchymal stem cells (MSC) prevented the progression of renovascular hypertension, improved renal function and architecture. PLoS One 8(11): e78464. 